PROCEEDINGS OF THE

AMERICAN MATHEMATICAL SOCIETY

Volume 126, Number 11, November 1998, Pages 3381-3383

S 0002-9939(98)04449-9

\title{
THE BAIRE CATEGORY THEOREM AND THE EVASION NUMBER
}

\author{
MASARU KADA \\ (Communicated by Carl G. Jockusch)
}

\begin{abstract}
In this paper we prove that $\mathfrak{e} \leq \operatorname{cov}(\mathcal{M})$ where $\mathfrak{e}$ is the evasion number defined by Blass. This answers negatively a question asked by Brendle and Shelah.
\end{abstract}

\section{INTRODUCTION}

Let $\operatorname{cov}(\mathcal{M})$ denote the smallest size of a family of meager sets whose union covers the real line. The combinatorial characterization for $\operatorname{cov}(\mathcal{M})$ has been studied by Miller and Bartoszyński, and the following result is established. For $g \in \omega^{\omega}$, let $\mathcal{S}^{g}=\prod_{n<\omega}[\omega] \leq g(n)$. Each element of $\mathcal{S}^{g}$ is called a slalom.

Theorem 1.1 ([1, Lemma 2.4.2]). The following cardinalities are the same:

1. $\operatorname{cov}(\mathcal{M})$.

2. the smallest size of $F \subseteq \omega^{\omega}$ such that for every $h \in \omega^{\omega}$ there exists $f \in F$ with $f(n) \neq h(n)$ for all but finitely many $n<\omega$.

3. the smallest cardinality $\kappa$ satisfying the following: for every $g \in \omega^{\omega}$ there exists $F \subseteq \omega^{\omega}$ of size $\kappa$ such that, for all $\varphi \in \mathcal{S}^{g}$, there exists $f \in F$ with $f(n) \notin \varphi(n)$ for all but finitely many $n<\omega$.

Blass [2] introduced a combinatorial concept called 'predicting and evading', and using this he defined the following cardinal invariant. Let $\mathcal{P}$ be the collection of functions $\pi$ from $\omega^{<\omega}$ to $\omega$. Here we call each such $\pi$ a predictor.

Definition $1.2([2])$. The evasion number $\mathfrak{e}$ is the smallest size of $F \subseteq \omega^{\omega}$ such that, for every $\pi \in \mathcal{P}$ and $X \in[\omega]^{\omega}$, there exists $f \in F$ with $f(n) \neq \pi(f\lceil n)$ for infinitely many $n \in X$.

Brendle and Shelah [3], [4] studied the relations between $\mathfrak{e}$ and other cardinal invariants, and asked whether $\mathfrak{e}>\operatorname{cov}(\mathcal{M})$ is consistent [4, Subsection 3.4]. Here we give a negative answer to this question.

Received by the editors February 10, 1997 and, in revised form, April 7, 1997.

1991 Mathematics Subject Classification. Primary 03E05.

The author was supported by JSPS Research Fellowships for Young Scientists. The author was also supported by Grant-in-Aid for Scientific Research (Encouragement for Research Fellow, No. 97-03909), Ministry of Education, Science and Culture.

(C) 1998 American Mathematical Society 


\section{THE MAIN RESULT}

We introduce a different form of evasion number by modifying the original definition due to Blass.

Definition 2.1. $\mathfrak{e}^{*}$ is the smallest size of $F \subseteq \omega^{\omega}$ such that, for every $\pi \in \mathcal{P}$ there exists $f \in F$ with $f(n) \neq \pi(f\lceil n)$ for all but finitely many $n<\omega$.

Clearly $\mathfrak{e} \leq \mathfrak{e}^{*}$ holds, and it is easily seen from Theorem 1.1 that $\operatorname{cov}(\mathcal{M}) \leq \mathfrak{e}^{*}$.

We show that $\mathfrak{e}^{*}$ gives another combinatorial characterization for $\operatorname{cov}(\mathcal{M})$. We prove the following theorem by modifying the proof for $\mathfrak{e} \leq \mathfrak{d}$, which is due to Blass $[2$, Theorem 13].

Theorem 2.2. $\mathfrak{e}^{*}=\operatorname{cov}(\mathcal{M})$.

Proof. For a function $h \in \omega^{\omega \times \omega}$, define $x_{h} \in \omega^{\omega}$ recursively so that $x_{h}(n)=$ $h\left(n, 1+\max \left\{x_{h}(i): i<n\right\}\right)$. Next, for a predictor $\pi \in \mathcal{P}$, define a function $\varphi_{\pi}$ from $\omega \times \omega$ to $[\omega]^{<\omega}$ by letting $\varphi_{\pi}(n, k)=\left\{\pi(t): t \in k^{n}\right\}$. By identifying $\omega \times \omega$ with $\omega$, we can regard $\varphi_{\pi}$ as a slalom in $\mathcal{S}^{g}$ for a suitable $g \in \omega^{\omega}$ which does not depend on $\pi$.

Now we prove the following: for $n<\omega$, if $h(n, k) \notin \varphi_{\pi}(n, k)$ for all $k$, then $x_{h}(n) \neq \pi\left(x_{h}\lceil n)\right.$. Suppose that $h(n, k) \notin \varphi_{\pi}(n, k)$ for all $k$. Let $k=1+$ $\max \left\{x_{h}(i): i<n\right\}$. Then $x_{h}\left\lceil n \in k^{n}\right.$ and hence $\pi\left(x_{h}\lceil n) \in \varphi_{\pi}(n, k)\right.$. On the other hand, $x_{h}(n)=h(n, k) \notin \varphi_{\pi}(n, k)$. Thus, $x_{h}(n) \neq \pi\left(x_{h}\lceil n)\right.$.

By Theorem 1.1, we can choose $F \subseteq \omega^{\omega \times \omega}$ of $\operatorname{size} \operatorname{cov}(\mathcal{M})$ so that, for each predictor $\pi \in \mathcal{P}$, there is $f \in F$ with $f(n, k) \notin \varphi_{\pi}(n, k)$ for all but finitely many $(n, k) \in \omega \times \omega$. Then the set $\left\{x_{f}: f \in F\right\}$ witnesses $\mathfrak{e}^{*} \leq \operatorname{cov}(\mathcal{M})$, and hence $\mathfrak{e}^{*}=\operatorname{cov}(\mathcal{M})$.

Corollary 2.3. $\mathfrak{e} \leq \operatorname{cov}(\mathcal{M})$.

Let $\operatorname{non}(\mathcal{M})$ denote the smallest size of a nonmeager set of reals. We can characterize non $(\mathcal{M})$ in a dual fashion, using [1, Lemma 2.4.8] instead of Theorem 1.1.

Theorem 2.4. $\operatorname{non}(\mathcal{M})$ is the smallest size of $\Pi \subseteq \mathcal{P}$ satisfying the following: for every $f \in \omega^{\omega}$ there exists $\pi \in \Pi$ such that $f(n)=\pi(f \uparrow n)$ for infinitely many $n<\omega$.

\section{A REMARK ON THE EVASION IDEAL}

Brendle [3, Subsection 3.5] introduced the notion of the evasion ideal, that is, the $\sigma$-ideal generated by the sets of the form

$$
\left\{f \in \omega^{\omega}: f(n)=\pi(f\lceil n) \text { for all but finitely many } n \in X\}\right.
$$

for $\pi \in \mathcal{P}$ and $X \in[\omega]^{\omega}$. He considered the smallest size of a subset of $\omega^{\omega}$ which does not belong to this ideal.

Definition 3.1 ([4, Definition 3.1]) $\mathfrak{e}(\omega)$, the uniformity of the evasion ideal, is the smallest size of $F \subseteq \omega^{\omega}$ satisfying the following: for any countable family of pairs $\left\{\left\langle\pi_{i}, X_{i}\right\rangle: i<\omega\right\} \subseteq \mathcal{P} \times[\omega]^{\omega}$ there is $f \in F$ such that for each $i<\omega$ we have $f(n) \neq \pi_{i}\left(f\lceil n)\right.$ for infinitely many $n \in X_{i}$.

Clearly $\mathfrak{e} \leq \mathfrak{e}(\omega)$ holds. Brendle and Shelah asked whether $\mathfrak{e}=\mathfrak{e}(\omega)$ can be proved in $\mathrm{ZFC}$, and they presented the following partial answer. 
Theorem $3.2([4$, Theorem 3.3]). $\mathfrak{e} \geq \min \{\mathfrak{e}(\omega), \operatorname{cov}(\mathcal{M})\}$. Thus either $\mathfrak{e}<$ $\operatorname{cov}(\mathcal{M})$ or $\mathfrak{e}(\omega) \leq \operatorname{cov}(\mathcal{M})$ implies $\mathfrak{e}=\mathfrak{e}(\omega)$.

We show that the latter assumption of the above theorem holds in ZFC.

Theorem 3.3. $\mathfrak{e}(\omega) \leq \mathfrak{e}^{*}$.

Proof. Fix $F \subseteq \omega^{\omega}$ of size less than $\mathfrak{e}(\omega)$ arbitrarily. Then we can choose a countable set of pairs $\left\{\left\langle\pi_{i}, X_{i}\right\rangle: i<\omega\right\} \subseteq \mathcal{P} \times[\omega]^{\omega}$ so that, for every $f \in F$, there is $i<\omega$ such that $f(n)=\pi_{i}\left(f\lceil n)\right.$ for all but finitely many $n \in X_{i}$. By shrinking $X_{i}$ 's if necessary, we can assume that $X_{i}$ 's are pairwise disjoint. Now define $\pi \in \mathcal{P}$ as follows: for $t \in \omega^{<\omega}$ if $|t| \in X_{i}$ for some $i<\omega$ then $\pi(t)=\pi_{i}(t)$; otherwise $\pi(t)$ is arbitrary. Then for all $f \in F$ we have $f(n)=\pi(f\lceil n)$ for infinitely many $n<\omega$.

Corollary 3.4. $\mathfrak{e}=\mathfrak{e}(\omega)$.

Proof. By Theorems 2.2, 3.2 and 3.3.

\section{ACKNOWLEDGEMENT}

I would like to thank the referee for his helpful comment, which gives another view of the main theorem I had not noticed.

\section{REFERENCES}

[1] T. Bartoszyński and H. Judah, Set theory: On the structure of the real line, A. K. Peters, Wellesley, Massachusetts, 1995. MR 96k:03002

[2] A. Blass, Cardinal characteristics and the product of countably many infinite cyclic groups, J. Algebra 169 (1994), 512-540. MR 95h:20069

[3] J. Brendle, Evasion and prediction - the Specker phenomenon and Gross spaces, Forum Math. 7 (1995), 513-541. MR 96i:03042

[4] J. Brendle and S. Shelah, Evasion and prediction II, J. London Math. Soc. 53 (1996), 19-27. MR 97d:03061

Osaka Prefecture University, Sakai, Osaka, 599-8531 Japan

E-mail address: kada@mi.cias.osakafu-u.ac.jp 